\title{
A food ingredient containing phytoecdysteroids and polyphenols from quinoa grain: technology and physiological and biochemical evaluation in vivo
}

\author{
Nikita Petrov*, Nadezhda Biryulina, Yuliia Sidorova, and Vladimir Mazo
}

Federal research centre of food and biotechnology, Department of food biotechnologies and specialized products, 109240, Ustiinskiy proezd, 2/14, Moscow, Russia

\begin{abstract}
An effective technological approach to produce adaptogenic microingredients is to concentrate plant biologically active substances via sorption on various matrices. The aim was to develop and evaluate in vivo the phytoecdysteroids and flavonoids concentrate extracted from quinoa grain and sorbed on the coagulated chicken egg protein. The consecutive extraction of phytoecdysteroids and flavonoids was conducted followed by sorption on the protein. The in vivo experiment was performed during 37 days using Wistar male rats. The developed concentrate was added into the diet of experimental animals in the dose $0.59 \pm 0.02 \mathrm{~g} / 100 \mathrm{~g}$ of diet. As a stress model we used daily 40-min immobilization and exhaustive 3-hour immobilization at the end of the experiment. Urinary catecholamines content was determined, blood corticosterone, malon dialdehyde, glutathione reductase and superoxide dismutase content was determined. Phytoecdysteroids and flavonoids were concentrated 20 and 80 times respectively compared to the initial raw materials. The concentrate consumption neutralized negative effect of immobilization stress on anxiety level of Wistar rats. The significant decrease in urinary excretion of adrenaline and noradrenaline shows the pronounced adaptogenic effect of the concentrate, whereas phytoecdysteroids and flavonoids act as prostressors activating body antioxidant protection system, what is substantiated by significantly increased blood superoxide dismutase level of experimental animals.
\end{abstract}

\section{Introduction}

One of the most important factors contributing to the formation of nonspecific resistance of the body to various adverse stressful influences is a healthy nutrition that meets the energy needs of the body and adequately provides it with essential nutrients: macro and micronutrients and minor biologically active food substances. One of the most effective solutions to the problem of ensuring an increase in the adaptive potential of the human body is the creation of a wide range of specialized food products that meet high modern safety

\footnotetext{
* Corresponding author: petrov-nikita-y@mail.ru
} 
and quality requirements. Adaptogenic properties of developed specialized food products depend mostly on the microingredients in their composition - the complexes of minor biologically active substances characterized from the standpoint of evidence-based medicine.

Quinoa grain (Chenopodium quinoa) - the pseudocereal food plant - is the prospective source of flavonoids and 20-hydroxyecdysone (20E, major representative of phytoecdysteroids): the phytonutrients with pharmacological properties, including antioxidant and adaptogenic [1-3].

An effective technological approach to the production of microingredients with adaptogenic properties is to concentrate the minor biologically active substances from plant extracts via sorption on the various matrices, in particular coagulated chicken egg protein with high biological value [4].

The aim of this work was to obtain the concentrate of phytoecdysteroids and flavonoids extracted from quinoa grain and sorbed on the coagulated chicken egg protein and to conduct its physiological and biochemical evaluation in vivo using Wistar male rats.

\section{Material and methods}

Black quinoa grain was grinded using knife mill and sifted through the sieve with pores diameter $0.35 \mathrm{~mm}$. The extraction of $20 \mathrm{E}$ and flavonoids from grinded and sifted grain was conducted at a temperature $25^{\circ} \mathrm{C}$ during 1 hour by means of mixing $200 \mathrm{~g}$ of grains with $4000 \mathrm{~mL}$ of $40 \%$ ethanol. The mixture was centrifuged at $3000 \mathrm{rpm}$ during $30 \mathrm{~min}$, the supernatant was collected. The supernatant was ultrafiltered through the membrane with pore diameter $10 \mathrm{kDa}$ using micro- and ultrafiltration apparatus (Vladisart, Russia). The obtained ultrafiltrate was concentrated on the reverse osmosis apparatus (Vladisart, Russia). Ethanol was evaporated from the obtained extract using rotary evaporator. The content of $20 \mathrm{E}$ in the extract was $255.3 \pm 2.5 \mu \mathrm{g} / \mathrm{mL}$, the content of flavonoids was $477.3 \pm 1.1 \mu \mathrm{g} / \mathrm{mL}$.

The coagulated chicken egg protein was obtained by separation of chicken egg yolk, mixing of liquid protein mass, acidifying with citric acid with the addition of $\mathrm{NaCl}(0.13$ and $0.8 \%$ respectively), incubating at the temperature $25^{\circ} \mathrm{C}$ during $15 \mathrm{~min}$ followed by heat treatment at the temperature $60-70^{\circ} \mathrm{C}$ during $1-3$ min with the constant mixing till the structure of cottage cheese. After the separation of liquid phase the obtained coagulated protein was cooled, lyophilized and powdered [4].

The concentrate of phytoecdisteroids (20E) and flavonoids complex extracted from grain was obtained via its sorption on the coagulated chicken egg by mixing the protein and the extract in the ratio $1 \mathrm{~g}$ of protein $/ 45 \mathrm{~mL}$ of extract at $25^{\circ} \mathrm{C}$ for 1 hour followed by lyophilization.

The content of $20 \mathrm{E}$ in the samples was determined by HPLC method using chromatograph Agilent Technologies 1100 (AgilentTechnologies, USA) with mass-detector Agilent Technologies 6410 using the column Agilent Technologies Poroshell 120 EC-C18 $3.0 * 50 \mathrm{~mm}, 2.7 \mu \mathrm{m}[5]$.

The total flavonoids content was determined by HPLC method (chromatograph Ultimate 3000 (Dionex, USA) with diode-matrix spectrophotometric detector and triple quadrupole mass-spectre detector TSQEndura (ThermoFisherScientific, USA)) using the column PhenomenexLunaC18 150*4.6 mm, $5 \mu \mathrm{m}$ [6].

The experimental batch of the developed concentrate was produced for the in vivo experiment. 


\subsection{Design of in vivo experiment}

The in vivo experiment was conducted using 36 male Wistar rats with initial body weight $130 \pm 5 \mathrm{~g}$. The experiment lasted 37 days. Animals were held 2 rats per cage in the controlled environment conditions (temperature $20-26^{\circ} \mathrm{C}$, relative humidity $30-60 \%$, 12-hour light cycle).

For the correct initial separation of animals into groups the parameters of animals anxiety were determined in the elevated plus maze test (EPM, Panlab, Spain) [7]. The test was conducted after 7-day quarantine: before the start of the experiment and on the $28^{\text {th }}$ day of the experiment to assess the changes in the animals anxiety level.

Animals were randomized according to the body weight and EPM results into three groups: $\mathrm{C} 1, \mathrm{G} 2$ and $\mathrm{G} 3(\mathrm{n}=12)$. Animals of the control $\mathrm{C} 1$ group and $\mathrm{G} 2$ group received for 36 days a standard half-synthetic diet [8]. Rats of the experimental G3 group received modified half-synthetic diet with the addition of phytoecdysteroids and flavonoids concentrate $(0.59 \pm 0.02 \mathrm{~g} / 100 \mathrm{~g}$ of diet). All diets were isocaloric $(376 \pm 2 \mathrm{kcal})$ and isonitrogenous.

Animals received food and water ad libitum, food intake was registered in a day. Animals were weighed two times a week. On the $22^{\text {nd }}$ day of the experiment animals were tested in the Open Field test [9].

Rats from G2 and G3 groups were exposed to daily 40-min immobilization for 35 days via putting into the transparent locking houses, restricting animals from free move.

On the $36^{\text {th }}$ day of the experiment animals of G2 and G3 groups were exposed to exhaustive 30hour immobilization. Immediately after the immobilization animals were moved to exchange cages to collect daily urine.

On 24 hours ( $37^{\text {th }}$ day of the experiment) preliminary deprived rats were decapitated under light ether anesthesia. Blood was collected: for plasma into the tubes with preliminary added anticoagulant; for serum into the empty tubes. Samples were centrifuged at $4000 \mathrm{rpm}$ for $15 \mathrm{~min}$ and stored at $-20^{\circ} \mathrm{C}$.

The catecholamines content: noradrenaline, adrenaline and dopamine in the urine was determined using liquid chromatograph Agilent 1100 (Agilent Technologies, USA). The blood plasma corticosterone content was determined by means of competitive ELISA commercial kit (IDS Limited, UK). To evaluate the condition of antioxidant system blood levels of superoxide dismutase, glutathione reductase and malondialdehyde were determined. The content of superoxide dismutase was determined using commercial analytical kit (Cayman Chemical, USA) and the contetnts of glutathione reductase and malondialdehyde were determined using commercial ELISA kits (Cloud-Clone Corporation, USA).

\subsection{Statistical data analysis}

The statistical analysis of obtained results was performed using SPSS Statistics 20 software (IBM, USA). Non-parametric T-test and Mann-Whitney test were used. Average value (M), standard deviation (SD) and standard error $(\mathrm{m})$ were calculated. Data are presented as $\mathrm{M} \pm \mathrm{m}$.

\section{Results and discussion}

The content of $20 \mathrm{E}$ in the grinded quinoa grain was $0.16 \pm 0.02 \mathrm{mg} / \mathrm{g}$, the content of flavonoids was $0.19 \pm 0.01 \mathrm{mg} / \mathrm{g}$.

The obtained concentrate of phytoecdysteroids and flavonoids, extracted from quinoa, represented finely divided light-brown powder. The content of $20 \mathrm{E}$ in the obtained product 
was $3.4 \pm 0.3 \mathrm{mg} / \mathrm{g}$, the content of flavonoids was $14.1 \pm 0.1 \mathrm{mg} / \mathrm{g}$. The protein content in the composition of concentrate was $98 \%$.

\subsection{Results of in vivo experiment}

The general condition of all animals in appearance, fur quality, food intake, water consumption and behavior during daily examination throughout the experiment was satisfactory.

Average food intake of stressed animals of G2 (17.2 $\pm 0.3 \mathrm{~g} / \mathrm{day} / \mathrm{rat})$ and G3 $(16.9 \pm 0.2$ $\mathrm{g} /$ day/rat) groups was significantly $(\mathrm{p}<0.05)$ lower in comparison with food intake of $\mathrm{C} 1$ $(19.4 \pm 0.3 \mathrm{~g} / \mathrm{day} / \mathrm{rat})$ group animals. The consumption of $20 \mathrm{E}$ in the composition of phytoecdysteroids and flavonoids concentrate by G3 group animals was $1.53 \pm 0.02 \mathrm{mg} / \mathrm{kg}$ b.w./ day, for flavonoids the value was $6.20 \pm 0.10 \mathrm{mg} / \mathrm{kg}$ b.w./day.

Starting from the $8^{\text {th }}$ day of the experiment the body weight of G2 and G3 group animals exposed to chronic immobilization stress was significantly $(\mathrm{p}<0.01)$ lower compared to body weight of $\mathrm{C} 1$ group animals. Immobilization has strong stress impact on the Wistar male rats and leads to significant decrease in animals body weight [10].

During the first test on the EPM (day 0), no significant differences were found in the time intervals spent by animals of all groups in the open (OA) and closed (CA) arms of the labyrinth (indicators characterizing the anxiety of animals); no significant differences in research activity, characterized by indicators of movement between the arms of the labyrinth and the total distance traveled, were also not revealed between the compared groups at the beginning of the experiment.

During the second test on the $28^{\text {th }}$ day of the experiment the behaviour of animals has changed. Animals of $\mathrm{C} 1$ group spent significantly less time in OA of labyrinth and significantly more time in CA of labyrinth in comparison with the first test $(\mathrm{p}<0.01)$, what is consistent with age-related alterations of animals behaviour. At that, animals of G2 and G3 groups spent significantly more time in OA of the labyrinth and significantly less time in CA of the labyrinth in comparison with $\mathrm{C} 1$ group animals $(\mathrm{p}<0.01)$. The similar result was also obtained for the indices of total exploration activity: total distance and the number of transitions between arms of the labyrinth of G2 group animals were significantly higher ( $\mathrm{p}<0.01)$ in comparison with $\mathrm{C} 1$ group animals. The total distance of G3 group animals, treated with phytoecdysteroids and flavonoids concentrate, was significantly higher compared to the animals of $\mathrm{C} 1$ group $(\mathrm{p}<0.01)$.

Figure 1 (A.B.C.D.E.F.) shows the results of the Open field test after 22 days of feeding of animals with experimental diets. 

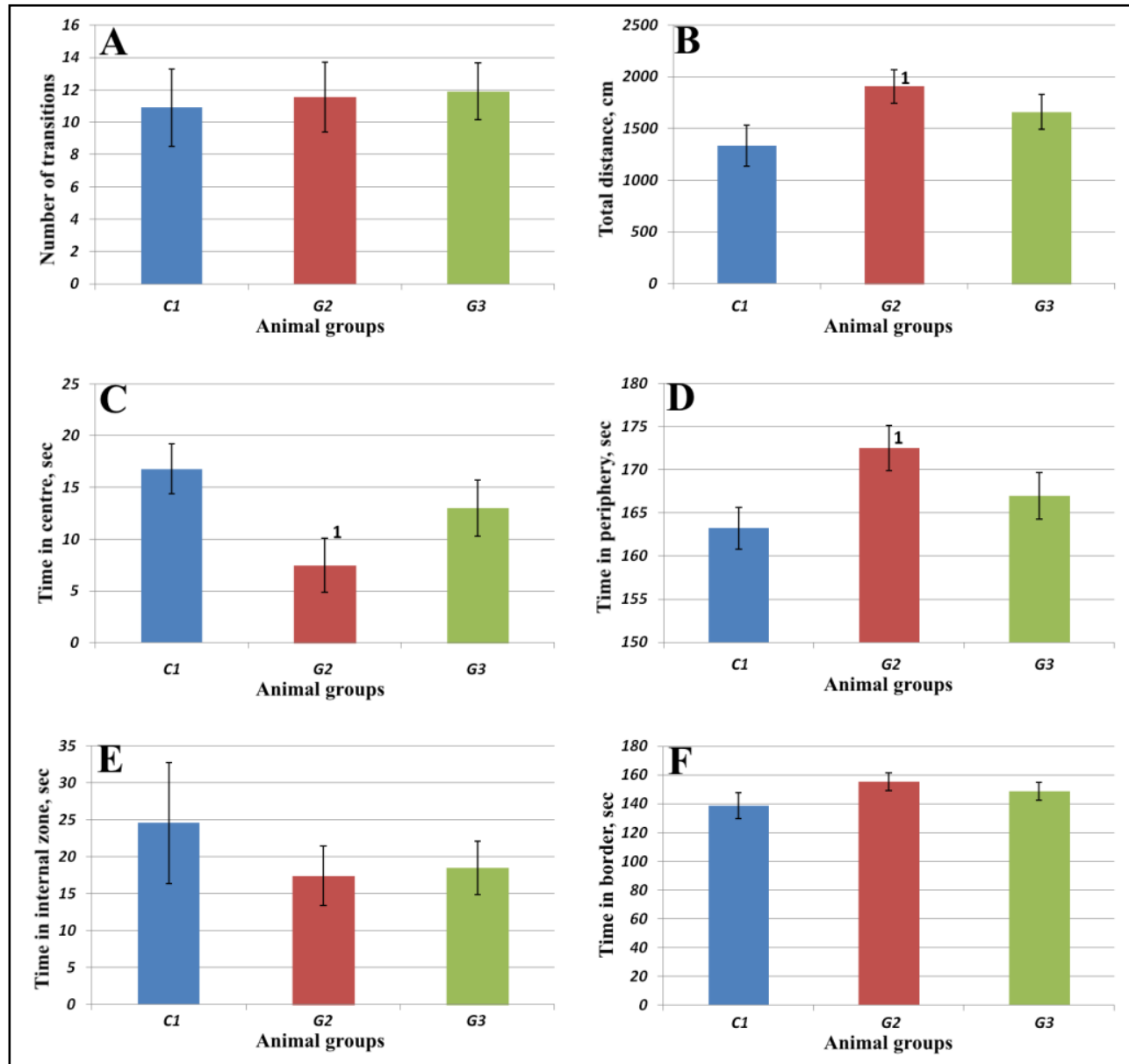

Note: 1- differences are significant in comparison with $\mathrm{C} 1$ group $(\mathrm{p}<0.05)$.

Fig. 1. Open field test results

In the Open Field test, there were no significant differences in the behaviour of the animals of the G3 group, which received the phytoecdysteroids and flavonoids concentrate, compared with the animals of the $\mathrm{C} 1$ group. Animals of the G2 group spent significantly less time in the centre of labyrinth and significantly more time in the peripheral zone of the labyrinth (Fig. 1. C.D.E.F.) in comparison with $\mathrm{C} 1$ group animals $(\mathrm{p}<0.05)$, which shows that animals exposed to immobilization has higher anxiety. However, these animals moved significantly more (Fig. 1.B.) in comparison with animals of $\mathrm{C} 1$ group $(\mathrm{p}<0.05)$, what is in accordance with the results of EPM test. There were no significant differences in anxiety and exploration activity between animals of G2 group and G3 group, receiving phytoecdysteroids and flavonoids concentrate. The obtained result may show that the consumption of phytoecdysteroids and flavonoids concentrate, sorbed on coagulated chicken egg protein, partially neutralized negative effect of immobilization stress on anxiety level of experimental animals.

Figure 2 shows the daily urinary excretion of catecholamines. 

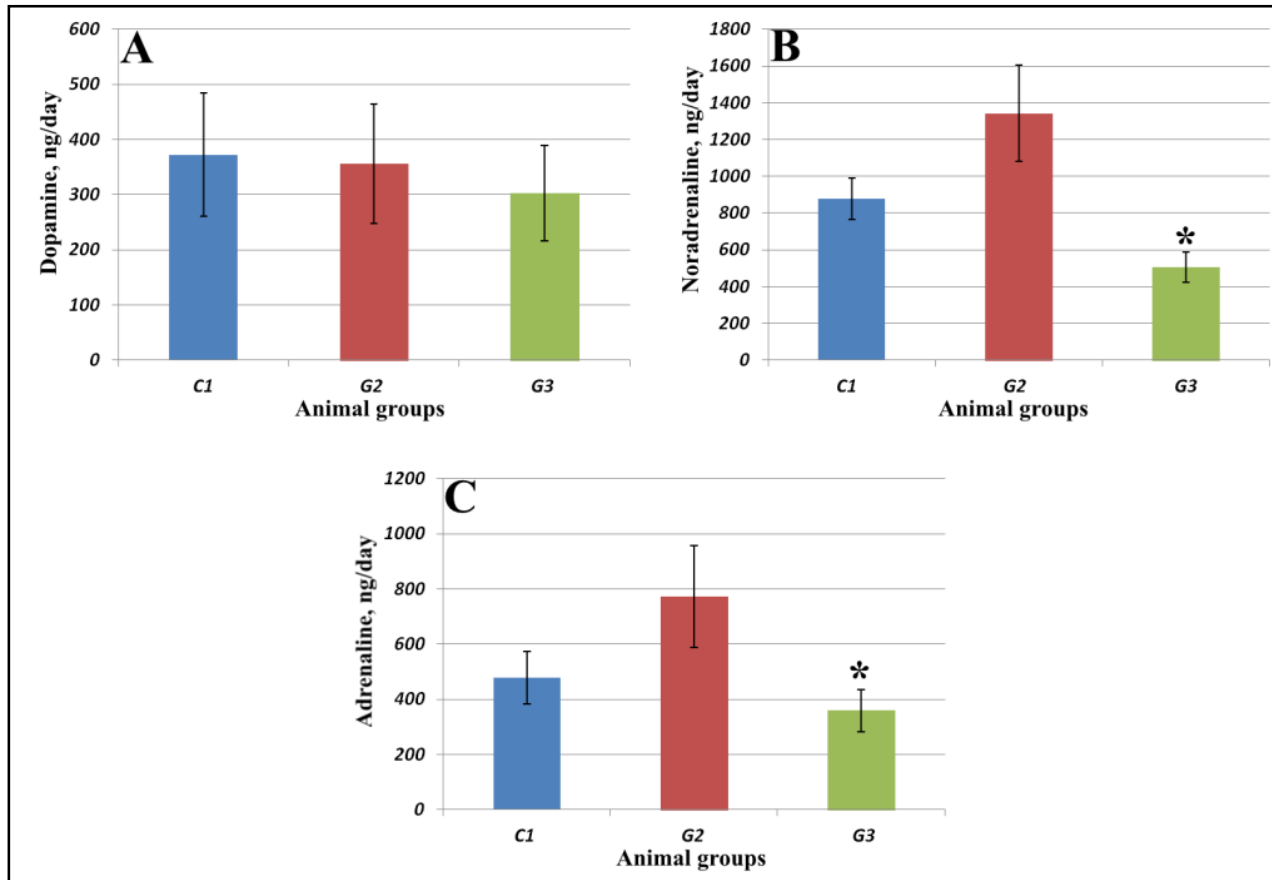

Note: * - differences are significant in comparison with G2 group, $\mathrm{p}<0.05$.

Fig. 2. Daily urinary excretion of catecholamines, ng/day

There were no significant differences in the daily urinary dopamine excretion in animals of all groups. At the same time, the daily urinary excretion of noradrenaline and adrenaline in the G3 group animals, which received the phytoecdysteroids and flavonoids concentrate, was significantly lower than in the animals of the G2 group ( $p<0.01)$. A decrease in urinary excretion of one of the main markers of the stress system indicates the adaptogenic effect of a phytoecdysteroids and flavonoids concentrate sorbed on the coagulated chicken egg protein.

Convincing evidence of the stressful effect of forced daily immobilization was a significant increase in the level of corticosterone in the blood plasma in rats of the G2 $(48.2 \pm 10.5 \mathrm{ng} / \mathrm{mL})$ and $\mathrm{G} 3(45.9 \pm 9.7 \mathrm{ng} / \mathrm{mL})$ groups as compared with the animals of the C1 group $(19.2 \pm 4.4 \mathrm{ng} / \mathrm{mL}, \mathrm{p}<0.05)$. The introduction of a phytoecdysteroids and flavonoids concentrate into the diet did not have any compensatory effect on the level of corticosterone, one of the main biomarkers of stress in the animals blood.

Oxidative stress is one of the pathological conditions that form under strong stressful effects [11]. Some biochemical indicators characterizing the antioxidant status of the animal body are presented in Table 1.

Table 1. Biochemical parameters of the antioxidant status

\begin{tabular}{|l|c|c|c|}
\hline \multirow{2}{*}{ Blood plasma parameters } & \multicolumn{3}{|c|}{ Animal group } \\
\cline { 2 - 4 } & C1 & G2 & G3 \\
\hline Malondialdehyde, $\mathrm{mg} / \mathrm{mL}$ & $9.7 \pm 1.5$ & $\mathbf{1 7 . 2} \pm \mathbf{3 . 6}^{\mathbf{1}}$ & $\mathbf{1 6 . 8} \pm \mathbf{3 . 0}$ \\
\hline Glutathione reductase, $\mathrm{ng} / \mathrm{mL}$ & $113.2 \pm 10.5$ & $93.6 \pm 7.0$ & $80.3 \pm 9.6$ \\
\hline $\begin{array}{l}\text { Superoxide dismutase, } \\
\text { units/mL }\end{array}$ & $1755.7 \pm 92.9$ & $1997.1 \pm 236.5$ & $\mathbf{2 5 0 5 . 3} \pm \mathbf{3 6 0 . 4}^{\mathbf{1}}$ \\
\hline
\end{tabular}

Note: 1 - differences are significant in comparison with $\mathrm{C} 1$ group, $\mathrm{p}<0.05$. 
The amount of malondialdehyde increased by more than $77 \%$ in rats of all groups exposed to immobilization stress compared to the animals of $\mathrm{C} 1$ group. The introduction of phytoecdysteroids and flavonoids concentrate into the diet of G3 group animals did not have any effect on the level of this parameter. There were no significant differences in the level of glutathione reductase between animals of groups.

The SOD activity in the blood serum of the G2 group animals in 24 hours after immobilization did not differ from the SOD activity in the blood serum of the $\mathrm{C} 1$ group animals. Our data are consistent with the results of work [12], which showed an increase in SOD activity in the blood serum of rats in 20 minutes after immobilization stress and its decrease after 24 hours to the control level. The animals of the G3 group showed a significant $(p<0.05)$ increase in SOD activity by $43 \%$ compared to the animals of the C1 group 24 hours after stress. An overabundance of superoxide usually indicates a high tension of the body's antistress system in an attempt to maintain homeostasis in animals after stress.

The increase in SOD activity revealed in our study 24 hours after stress exposure against the background of consumption of phytoecdysteroids and flavonoids concentrate by animals of the G3 group suggests that the main components of the concentrate (20hydroxyecdysone and flavonoids) can themselves act as prostressors and activate the antioxidant defense system.

Thus, this work presents a technological scheme for obtaining a concentrate of a complex of phytoecdysteroids and flavonoids extracted from quinoa grain sorbed on coagulated chicken egg protein. This technological scheme makes it possible to concentrate phytoecdysteroids more than 20 times, and flavonoids more than 80 times compared to the initial raw material. In an in vivo experiment, it was shown that the consumption of a phytoecdysteroids and flavonoids concentrate can neutralize the negative effect of immobilization stress on anxiety of Wistar rats. A significant decrease in urinary excretion of the main stress markers - adrenaline and noradrenaline - indicates a pronounced adaptogenic effect of the concentrate, while phytoecdysteroids and flavonoids act as prostressors, activating the body's antioxidant defense systems, which is confirmed by a significant increase in the level of superoxide dismutase compared to the control and stressed groups.

This work is supported by Russian Science Foundation (project № 19-16-00107).

\section{References}

1. Y. Tang, R. Tsao Mol. Nutr. Food Res. 61, 7 (2017)

2. V. Melini, F. Melini. Foods. 10, 2 (2021)

3. E.S. Ong, C.J.N. Pek, J.C.W. Tan, C.H. Leo. Antioxidants (Basel). 9, 11 (2020)

4. Yu.S. Sidorova, V.K. Mazo, S.N. Zorin, I.L. Stefanova. Voprosy pitaniia. 87, 1 (2018)

5. S.R. Yoo, S.J. Jeong, N.R. Lee, H.K. Shin, C.S. Seo. Pharmacogn Mag. 13, 51 (2017)

6. J.S. Park, I.S. Kim, S.U. Rehman, C.S. Na, H.H. Yoo. J Chromatogr Sci. 54, 2 (2016)

7. L. Castanheira, M.F. Ferreira, A.M. Sebastião, D. Telles-Correia. Curr. Top. Med. Chem. 18, 19 (2018)

8. P.G. Reeves. J Nutr. 127, 5 (1997)

9. P. Knight, R. Chellian, R. Wilson, A. Behnood-Rod, S. Panunzio, A.W. Bruijnzeel. Pharmacol Biochem Behav. 204, 173168 (2021)

10. Yu.S. Sidorova, N.A. Petrov, S.N. Zorin, V.K. Mazo. Bulletin of experimental biology and medicine. 171, 3 (2021) 
11. A. Banagozar Mohammadi, M. Torbati, F. Farajdokht, S. Sadigh-Eteghad, S.M.B. Fazljou, S.M. Vatandoust, S.E.J. Golzari, J. Mahmoudi. Brain Res. 1715, 47-56 (2019)

12. A.V. Pritvorova, A.V. Vyushina, N.E. Ordyan. Tendencies in the science and education development. 36-2, 61-63 (2018) 